between SSRs, as demonstrated through simulations and measurements. The researchers conclude that by combining two SRR metamaterials, one with negative $\mu$ and the other with negative $\varepsilon$, a 3D negative index material could be achieved. In addition to the media-favorite of invisibility cloaking, such a material could find application in optical computing, nanoscale imaging, and high-speed communications.

Alison HatT

\section{Atomistic Approach Predicts Resonance Frequency of Viral Capsids for Mechanodestruction Treatment}

Viruses cause many severe diseases for humans, animals, and plants, adversely affecting public health and agricultural production. Treatment options for viral infections are typically based on drug chemotherapies or vaccines, which often lead to negative side effects. Furthermore, mutations in the virus structure may make vaccines ineffective. Recently, researchers have proposed to attack viruses not through chemical but through mechanical means, by using hypersound, T-rays, or light scattering radiation. These methods are based on the idea to excite lowfrequency mode vibrations at their resonance frequency, which leads to rupture of the viral capsid structure and thereby a destruction of the virus. A similar phenomenon is known at macroscopic scales from bridges and buildings, which may suddenly collapse if excited at their resonance frequencies by wind loads. In order to optimally apply this new treatment option, it is desirable to know the resonance frequencies of the virus structure.

How can one predict these resonance frequencies, provided the atomic structure of the virus is known? In the January 18 issue of Physical Review Letters (028101; DOI: 10.1103/PhysRevLett.100.028101), E.C. Dykeman and O.F. Sankey of Arizona State University describe a new computational method to predict the low-frequency vibrational modes of biological structures based on their atomistic geometry. The new approach is based on an energy functional from electronic structure theory and provides a much more efficient strategy to analyze the atomistic properties of virus vibrations than earlier attempts. The method is robust and is now being applied to plant viruses (e.g., cowpea chlorotic mottle virus), bacteriophages (e.g., M13 bacteriophage), and human viruses (e.g., polio and hepatitis B).

A significant advantage of Dykeman and Sankey's approach is that it allows for an all-atom description of the vibrational mode patterns based on the atomistic structure of the virus, which can be obtained from an x-ray diffraction analysis of the virus geometry.

Using the new approach, Dykeman and Sankey calculated the vibrational modes of the satellite tobacco necrosis virus based on the atomistic geometry obtained from an earlier $x$-ray diffraction analysis. Their calculations result in the prediction of the specific resonance frequencies of excitation that will most likely lead to a destruction of the virus. It also provides insight into the particular molecular deformation mechanisms, such as shearing of beta-sheets. This new tool could be an important computational tool in developing new treatment options for newly discovered types of viruses, according to the researchers.

This theoretical group works in close collaboration with an experimental effort led by colleague K.-T. Tsen of Arizona State, who has had success in inactivating viruses by inelastic light scattering. Future studies may include experimental verification of the predicted vibrational frequencies of specific viruses.

MARKUS J. BUEHLER

\section{CNT FET with Asymmetric Contact Shows Single Electron Transistor Characteristics at Low \\ Temperature}

Recent studies have shown that carbon nanotube (CNT) room-temperature single electron transistors (RTSETs) have been obtained by manipulation with an atomic force microscope (AFM) and by local chemical modification. However, almost all reported studies on RTSETs are with symmetric drain (D) and source (S) electrodes. Now, H. Li and Q. Zhang from Nanyang Technological University, Singapore and N. Marzari from the Massachusetts Institute of Technology have reported on a type of short singlewalled CNT (SWCNT)-field-effect transistor (FET), which shows coulomb blockade effects with asymmetric D and S electrodes at low temperatures. Moreover, the devices exhibit current rectification characteristics at room temperature.

In the January issue of Nano Letters (p. 64; DOI: 10.1021/nl071905e), the researchers report the fabrication of SWCNT-FETs with a channel length of $~ 90$ $\mathrm{nm}$. The researchers started with a highly boron-doped silicon wafer, which was capped by thermal oxide of $500 \mathrm{~nm}$. Their interdigital electrode of $\mathrm{Au} 90 \mathrm{~nm} / \mathrm{Ti}$ $10 \mathrm{~nm}$ thickness was patterned on this thermal oxide. SWCNTs were placed across these electrodes by a dielectrophore- sis technique. They specifically kept the concentration of the SWCNT suspension very low in order to have only a few SWCNTs bridged between these interdigital electrodes. The $\mathrm{Al}$ (source) was deposited in a way so that one end of the SWNTchannel was attached to the sidewall of the $\mathrm{Au}$ (drain) electrodes and the other end was buried beneath the deposited $\mathrm{Al}$ layer in order to create a shallow gap between the $\mathrm{Al}$ and $\mathrm{Au}$ electrodes. At room temperature, they found that the device shows diode-like characteristics with a maximum current rectification ratio of $\sim 10^{4}$. But at $25 \mathrm{~K}$, they found a diamond-shaped current suppression region in the contour plot of the drain-source current mapped in a plane as a function of the drain-source bias voltage (on one axis) and the gate-source voltage bias (on the other axis), which they identified as the Coulomb blockade effect that is usually found in single electron transistors. With increasing temperature, the thermal energy becomes comparable to or even larger than the Coulomb charging energy. As a result, the oscillation peaks broaden and fade away when the temperature is $\sim 100 \mathrm{~K}$.

The researchers concluded that due to the unequal thermionic emission current in the asymmetric contacts (as a result of their different barrier heights), they saw asymmetric current amplitudes under positive and negative drain-source voltage bias. The barrier height for electrons at the $\mathrm{Au} / \mathrm{SWCNT}$ contact is larger than that at the Al/SWCNT contact by a factor of $>2$ for all gate voltages. In an atmosphere environment, the device shows significant diode-like effects with maximum current rectification ratios of $>10000$.

M. GOWTHAM

\section{Slithering of Shear Bands Can Improve Plasticity in Bulk Metallic Glasses}

Glasses have evolved through many developments, starting from Obsidian (a natural glass) to metallic glasses and bulk metallic glasses (BMGs). BMGs have been developed since the 1990s as a new class of engineering materials, which offer an opportunity to revolutionize the field of structural materials with an excellent combination of ductility, strength, and toughness.

Failure of BMGs under extensive deformation through the formation of shear bands at room temperature has been well documented for $\mathrm{Pt}-, \mathrm{Cu}-, \mathrm{Pd}, \mathrm{Ti}-$, and $\mathrm{Zr}$ based glassy alloys. However, this disadvantage has been overcome by the recent development of unique ductile BMGs.

As described in the January issue of the 